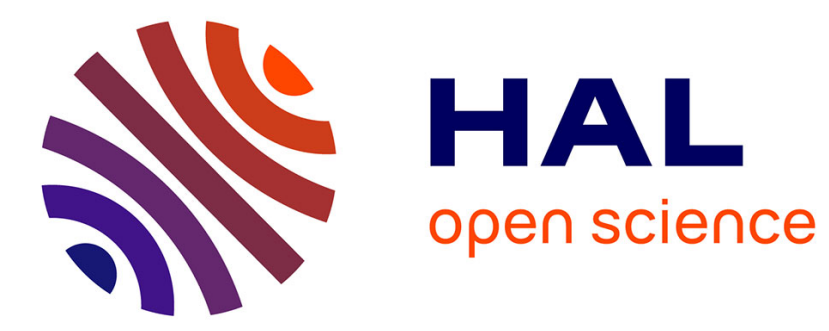

\title{
Robust Aircraft Optimal Trajectory in the Presence of Wind
}

Karim Legrand, Stéphane Puechmorel, Daniel Delahaye, Yao Zhu

\section{To cite this version:}

Karim Legrand, Stéphane Puechmorel, Daniel Delahaye, Yao Zhu. Robust Aircraft Optimal Trajectory in the Presence of Wind. IEEE Aerospace and Electronic Systems Magazine, 2018, 33 (11), pp.Pages 30-38. 10.1109/MAES.2018.170050 . hal-01759014

\section{HAL Id: hal-01759014 \\ https://hal-enac.archives-ouvertes.fr/hal-01759014}

Submitted on 5 Apr 2018

HAL is a multi-disciplinary open access archive for the deposit and dissemination of scientific research documents, whether they are published or not. The documents may come from teaching and research institutions in France or abroad, or from public or private research centers.
L'archive ouverte pluridisciplinaire HAL, est destinée au dépôt et à la diffusion de documents scientifiques de niveau recherche, publiés ou non, émanant des établissements d'enseignement et de recherche français ou étrangers, des laboratoires publics ou privés. 


\title{
Robust Aircraft Optimal Trajectory in the Presence of Wind
}

\author{
K.Legrand, ENAC, 7 Avenue Edouard Belin, 31055 Toulouse France (klegrand@recherche.enac.fr) \\ S.Puechmorel, ENAC, 7 Avenue Edouard Belin, 31055 Toulouse France (stephane.puechmorel@enac.fr) \\ Daniel DELAHAYE, ENAC, 7 Avenue Edouard Belin, 31055 Toulouse France (delahaye@recherche.enac.fr) \\ Y.Zhu, ENAC, 7 Avenue Edouard Belin, 31055 Toulouse France (yaozhu537@gmail.com)
}

\begin{abstract}
Aircraft optimal trajectory planning in the presence of wind is a critical issue for airlines to save fuel. Planning is difficult due to the uncertainties linked to wind. Based on wind predictions, airlines have to compute trajectory planning for their aircraft in an efficient way. Such planning has to propose robust solutions which take into account wind variability.

In this paper, we propose a robust wind optimal trajectory design algorithm based on two phases. The first phase considers the wind map predictions and computes for each of them the associated wind optimal trajectory also called geodesic. Such geodesics are computed with a Bellman algorithm on a grid covering an elliptical shape projected on the sphere.

The second phase of the algorithm extract the most robust geodesic trajectories by the mean of a new trajectory clustering algorithm. This clustering algorithm is based on a new mathematical distance involving continuous deformation approach applied to north Atlantic flights ${ }^{1}$.
\end{abstract}

\section{INTRODUCTION}

With improvement of the environmental awareness, airlines have paid attention to reduce fuel consumption during daily flight operations. Airlines pursue to minimize the adverse effects of headwinds, or maximize the beneficial effect of tailwinds when planning flight trajectories. According to the jet stream profile of the world, it is easy to notice that the jet streams are in the east-west direction instead of the north-south direction. Therefore, the flights of east-west routes are affected more by en-route winds than the flights of north-south routes. In order to achieve the best flight performance in terms of the flight time and the fuel consumption, airlines may adjust the flight trajectories based on en-route wind profiles. Consequently, it is necessary to consider en-route wind effects when planning flight trajectories. However, it is difficult to identify the most suitable trajectory in a complex wind field mainly because such field is not easy to estimate [17], [25], [8], [9], [26]. The wind directions and strength are varying in different regions, at different altitudes and different times.

\footnotetext{
${ }^{1}$ This paper is an invited paper as a DASC best student paper.
}

Even though the problem is complex to solve, it may benefit airlines in terms of fuel cost and on-time performance.

Planning optimal trajectories is a rich and dynamic research domain with many application areas like robotics, space or aviation [6], [2], [3]. Depending on the problems' needs, the issues are different in nature and so are the techniques used to solve them. Here, we are interested in finding the global optimal path in presence of currents in a two dimensional space. Several methods, such as Dijkstra algorithm [10] or A* algorithm [20], discretize the domain and work on the generated network to find the optimal path. These algorithms are very efficient but the computed solution is restricted to the network. Some others algorithms work on the continuous space. Those algorithms are based on front propagation methods such as Level Set methods, Fast Marching methods and Ordered Upwind methods. These different algorithms are developed by Sethian in [13]. In [5], Petres adapts the Fast Marching Method to path planning for Autonomous Underwater Vehicles taking into account underwater currents. However, his algorithm cannot be applied to vehicles featuring behaviors more complex than a linear reaction to currents. In [15], Alton uses the Ordered Upwind algorithm with the SemiLagrangian method to generate optimal trajectories. In [3] a straightforward wind computation approach is presented, which relies on the difference between the predicted motion of the aircraft and the real motion measured by sensors. This wind estimation is fully tactical.

However, trajectory uncertainties (related to weather conditions) can not be completely eliminated; therefore, there are discrepancies between actual and projected trajectories.

Usually, aircraft trajectories are optimized in order to minimize some criteria : fuel, time, etc... When such planning is done in presence of wind one must take also into account the robustness of the planned trajectory. As a matter of fact airlines prefer to fly less efficient trajectories in terms of fuel but with a higher robustness.

In this paper, we address this robust trajectory planning in presence of wind with some uncertainties. 
Weather forecast usually propose several possible situation by producing Ensemble Prediction. Ensemble Prediction Systems (EPS) are an approach to weather forecasting that has been adopted by the Numerical Weather Prediction centers in order to characterize and quantify the uncertainty inherent to prediction [14]. This prediction technique involves generating a representative sample of the possible future states of the atmosphere. This collection of individual forecasts, called members, is generated by modifying the initial conditions and/or the meteorological model equations or parameters [1], [4].

The paper is organized as follow. The first part describes the algorithm to design aircraft optimal trajectory in the presence of wind. The second part presents the clustering algorithm with a focus on a new mathematical distance between trajectories. The third part gives some results of the application of our algorithm to the oceanic airspace in order to generates robust aircraft optimal trajectories in the presence of wind.

\section{Wind Optimal TRAJECTORY COMPUTATION}

\section{A. Wind Grid Computation and Interpolation}

We consider a 3-degree of freedom point-mass model of a fixed-wing aircraft flying though the North Atlantic Ocean. As an assumption, only cruise part of the flight is considered not only for the simplicity purposes but also because the cruise part is the majority of the flight. Additionally, we assume that the aircraft is flying at constant flight level. In this paper, we do not take temperature issue into account. As a result, we note that based on those assumptions, more complex problems can be simulated by applying this methodology easily.

We compute the optimal trajectory based on the wind predictions with a classical Bellman algorithm. In order to use Bellman algorithm to solve the problem, we need first to build a wind grid which stores wind data information.

1) Generate the wind grid: We generate a grid of size $N \times M$ nodes on he North Atlantic Ocean. The area from latitude 30 to latitude 70 and from longitude -90 to longitude 10 is taken into account. Each integer latitude and longitude point is regarded as a node. In order to generate smooth trajectories, we divide each latitude and longitude into 10 boxes. As a result, a $400 \times 1000$ grid table is generated.

2) Wind data interpolation: Note that, the wind data only contain the information at integer latitude and longitude node and we need to have the information at all nodes. We use Shepard's Method[7] to do such interpolation.

Let $F(P)$ be a function of the point $P=(x, y)$ defined for all $P$ in the real plane $\mathbb{R}^{2}$, the value at point $P$ is the weighted average of the values at nearby 4 data point $P_{1}, P_{2}, P_{3}$ and $P_{4}$ (integer node). Denote the value of $F$ at $P_{i}$ by $F_{i}$ and

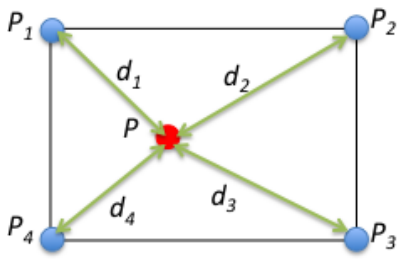

Fig. 1: Metric interpolation

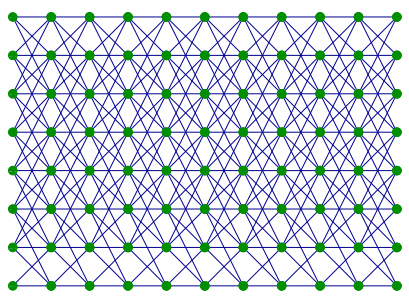

Fig. 2: Graph used for the wind optimal trajectory design.

$d_{i}$ be the distance between $P_{i}$ and the generic point $P$ in $\mathbb{R}^{2}$ (See Fig 1). The result was established by the function :

$$
F(P)=\left[\sum_{i=1}^{4} F_{i} \prod_{j \neq i} d_{j}\right] /\left[\sum_{i=1}^{4} \prod_{j \neq i} d_{j}\right]
$$

\section{B. Bellman Algorithm}

In order to generate wind optimal trajectories, we start building a graph $G=\{\mathcal{N}, \mathcal{L}\}$ based on the wind grid (see Fig 2), for which the set $\mathcal{N}$ represents the nodes and $\mathcal{L}$ the links.

Each node stores the following information : Latitude $\phi$, longitude $\lambda$, altitude $z$, the east wind component $W_{E}$ and the north wind component $W_{N}$. Based on those initial data coming from the wind grid, we compute also the wind norm $\|\vec{W}\|=\sqrt{W_{E}^{2}+W_{N}^{2}}$ at each node and the associated wind bearing $\theta_{W}$ (see Fig 3a).

The graph is structured into layers (see Fig 2), to improve the performance of the Bellman algorithm. As a matter of fact, thanks to this structure, only one Bellman algorithm iteration is needed to find the minimum path.

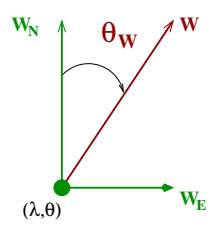

(a) Wind information contained in nodes

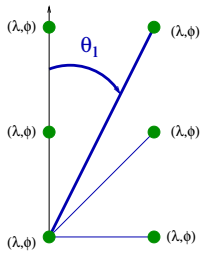

(b) Information contained in links
Fig. 3: Information in nodes and links. 


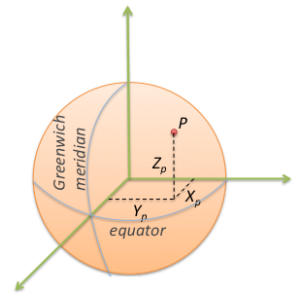

Fig. 4: The Cartesian coordinates

Each node has also a list of successive neighbors which are represented by the blue links on Fig 2. Each node (except the extreme north and extreme south) has some neighbors in the north direction and in the south direction (in our case, two in the north and two in the south). Such limitation will ensure smooth trajectory, avoiding sharp turns. Each link $l \in \mathcal{L}=\left(\mathcal{N}_{2}, \mathcal{N}_{C}\right)$ connects one origin node $N_{o}$ and one destination node $N_{d}$. The grand circle distance of link $l, d_{l}$ is given by the following formula :

$$
d_{l}=R \cdot \arcsin \left\{\left\|\vec{P}_{d} \wedge \vec{P}_{o}\right\|\right\}
$$

where $\vec{P}_{o}=\left(x_{o}, y_{o}, z_{o}\right)^{T} \vec{P}_{d}=\left(x_{d}, y_{d}, z_{d}\right)^{T}$ are the Cartesian coordinates of the nodes $N_{o}$ and $N_{d}, \wedge$ is the vector product and $R$ is the radius of the earth. For a given node $P$ (see Fig 4), the Cartesian coordinates are given by the following formula :

$$
\vec{P}=\left\{\begin{array}{l}
x=R \cdot \cos (\phi) * \cos (\lambda) \\
y=R \cdot \cos (\phi) * \sin (\lambda) \\
z=R \cdot \sin (\lambda)
\end{array}\right.
$$

where $\lambda$ is the latitude and $\phi$ is the longitude.

Each link contains also its associated bearing (see Fig $3 b$ ) $\theta_{l}$ which is given by the following formula :

$$
\left\{\begin{array}{l}
\theta_{l}\left(N_{o}, N_{d}\right)=\arctan \left(\frac{y}{x}\right) \\
y=\sin \left(\Delta_{\lambda}\right) \cdot \cos \left(\phi_{d}\right) \\
x=\cos \left(\phi_{o}\right) \cdot \sin \left(\phi_{d}\right)-\sin \left(\phi_{o}\right) \cdot \cos \left(\phi_{d}\right) \cdot \cos \left(\Delta_{\lambda}\right) \\
\Delta_{\lambda}=\lambda_{d}-\lambda_{o}
\end{array}\right.
$$

Based on the previous equation, one can now compute the tail wind on each extremities of the link $l\left(T W_{l o}\right.$ and $\left.T W_{l d}\right)$ :

$$
T W_{o}=\left\|\vec{W}_{o}\right\| \cdot \cos \left(\theta_{l}-\theta_{W_{o}}\right) T W_{d}=\left\|\vec{W}_{d}\right\| \cdot \cos \left(\theta_{l}-\theta_{W_{d}}\right)
$$

Those two tail winds are then averaged and associated to each link :

$$
T W_{l}=\frac{T W_{O}+T W_{d}}{2}
$$

This last tail wind will be used for the cost associated to each link in the shortest path computation.
To compute the wind optimal trajectory, we will consider for each link, the time needed by aircraft to connect node $N_{o}$ to node $N_{d}$. This time $t_{l}$ is given by :

$$
t_{l}=\frac{d_{l}}{T_{a}+T W_{l}}
$$

where $T_{a}$ is the true airspeed of the aircraft.

Having a graph with layer structure, a Bellman-Ford algorithm has been implemented for finding the shortest path between a node at the extreme left $\left(N_{o}\right)$ and all the nodes at the extreme right $\left(N_{d}\right)$. The algorithm is organized into three steps :

STEP 1: Initialization This step initializes distances (dist) from source to all vertices as infinite and distance to source itself as 0 .

STEP 2 : Propagation The source node is first considered and its associated neighboring links.

Starting from the source node $N_{O}=s r c$ (first column) and for each link associated to $N_{o}$ the algorithm marks the neighboring nodes of $N_{O}=s r c$ with the following rule ;

- if $\operatorname{dist}\left[N_{d}\right]>\operatorname{dist}\left[N_{o}\right]+d_{l}$ then update $\operatorname{dist}\left[N_{d}\right] \Rightarrow$ $\operatorname{dist}\left[N_{d}\right]=\operatorname{dist}\left[N_{o}\right]+d_{l}$ (keep in node $N_{d}$ the node $N_{o}$ which has been use for this update

Shift to the next column (column 2) and apply the same rule to all nodes which have been updated in order to propagate the distance update to the third column. This process is repeated until the propagation reach the last column (on the right).

STEP 3 : Path building To compute the shortest path for the source node src to any destination nodes on the right (dest), first select a destination node among the nodes belonging to the last column (column number $K$ ). Select the node $N_{K-1}$ in column $K-1$ which has updated the dest node in column $K$. Then, select the node $N_{K-2}$ in column $K-2$ which has updated the node $N_{K-1}$ in column $K-1$ and so on until the source node is reached in this back propagation process.

For each weather sample, such minimum time path algorithm is computed in order to create a set of trajectories that has to be clustered. To compute those trajectory clusters, a distance beetween trajectories has first to be defined.

\section{Trajectory Clustering Algorithm}

In order to group trajectories into clusters, one must first establish a mathematical distance between such mathematical objects.

\section{A. Mathematical Distance between Trajectories}

In a vector space, distances are very well defined.

For two points $\vec{P}_{1}=\left(x_{1}, y_{1}\right)^{T}$ and $\vec{P}_{2}=\left(x_{2}, y_{2}\right)^{T}$ in a plane (see Fig 5), the distance between them can be computed with the classical formula of the euclidean distance (see Fig 5). 

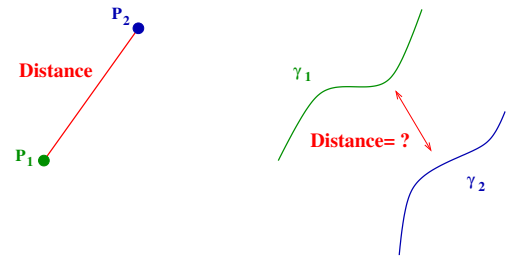

Fig. 5: On the left, two points $\vec{P}_{1}$ and $\vec{P}_{2}$ has been drawn for which the classical Euclidean distance is shown in red. On the right, two trajectories are drawn $\left(\gamma_{1}, \gamma_{2}\right)$ for which a mathematical distance has to be determined.

What is the distance, if now the points $\vec{P}_{1}$ and $\vec{P}_{2}$ are replaced by two trajectories $\gamma_{1}$ and $\gamma_{2}$ ? Trajectories are infinite dimension mathematical objects which are not easy to manipulate. One of the main results of this paper is the establishment of such mathematical distance.

1) Current Trajectory Distances: An aircraft trajectory is a time sequence of coordinates representing the aircraft path over a period of time and may be represented by a $N$ uple $: T=\left\{\left(x_{1}, y_{1}, z_{1}, t_{1}\right),\left(x_{2}, y_{2}, z_{2}, t_{2}\right), \ldots,\left(x_{N}, y_{N}, z_{N}, t_{N}\right)\right\}$ where $N$ is the duration.

The simplest metric used for computing the distance between a pair of trajectories is the mean of coordinate distances.

Note that, the mean of distance metric makes three critical assumptions :

1) the durations of both trajectories are the same : $N^{a}=$ $N^{b}=N$

2) the coordinates are synchronized $t_{n}^{a}=t_{n}^{b}$

3) the time sampling rate is constant $t_{n+1}^{a}-t_{n}^{a}=t_{m+1}^{a}-t_{m}^{a}$ It is evident that the mean of distance is very sensitive to the partial mismatches and cannot deal with the distortions in time.

To provide more descriptive information, the second order statistics such as median, variance, minimum and maximum distance may be incorporated.

Although these statistics supply extra information, they inherit (even amplify) the shortcomings of the ordinary mean of distance metric $m_{1}$. None of the above metrics is sufficient enough by itself to make an accurate assessment of the similarity.

Another possible candidate for the distance between two trajectories $\gamma_{1}$ and $\gamma_{2}$ will simply be to take the supremum norm $d_{\infty}\left(\gamma_{1}, \gamma_{2}\right)$ (see Fig 6).

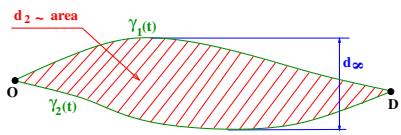

Fig. 6: Supremum norm distance

Since $\gamma_{1}$ and $\gamma_{2}$ are constant outside bounded intervals

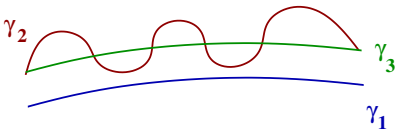

Fig. 7: Different trajectories with same sup distance. The sup norm between the green trajectory and the red trajectory is the same as the one computed between the green and the blue trajectories; meaning the such sup norm consider $\gamma_{1}$ and $\gamma_{2}$ at the same distance from $\gamma_{3}$ event if $\gamma_{1}$ and $\gamma_{2}$ are very different.

of $\mathbb{R}$, the supremum is well defined. However, this metric is not sensitive to global properties of curves. In the Fig 7, the curves $\gamma_{1}$ and $\gamma_{2}$ are at the same distance from $\gamma_{3}$ but have very different shapes.

From an operational point of view, $\gamma_{1}$ is just a shifted copy of $\gamma_{3}$ while $\gamma_{2}$ will probably not be realistic.

For trajectories $\gamma_{1}, \gamma_{2}$ with the same origin-destination pairs, $\gamma_{1}-\gamma_{2}$ can be defined as a compactly supported mapping and an area distance between trajectories $\left(d_{2}\left(\gamma_{1}, \gamma_{2}\right)\right)$ can be defined (see Fig 6).

This distance can handle more complex trajectories, however it is sensitive to entanglements of the trajectory, discards the time continuity, and fails to distinguish two trajectories in opposite directions. Furthermore, it can not be extended to the third dimension.

In order to introduce our new mathematical distance between trajectories, one must first give some representation definitions.

2) Representation: Since objects of interest are aircraft trajectories, we need to find an adapted framework in which computations may be made on trajectories as a whole. There are basically two ways of understanding what a trajectory is :

- The time/position approach for wich time is included in the representation. In this case, a trajectory can be represented as a mapping from a bounded interval of $\mathbb{R}$ (the life time of the trajectory) to $\mathbb{R}^{3}$ or $\mathbb{R}^{6}$ depending on whether speed is part of the data or not. Since there is an explicit dependence on time, there is a need to calibrate trajectories with time shifts for all applications involving trajectory comparison. We will see in the following that there is a mean of reducing the problem by calibrating automatically the origin of time.

- The shape approach for which only the path is considered without time. Here, trajectories are understood as paths and time is not directly relevant (from a more formal point of view, we take the quotient of the trajectories understood as mappings by the group of diffeomorphisms acting on time), so that we may assume that the underlying life time of trajectories is 
always the interval $[0,1]$. This framework is adpated for dealing with major flows estimation.

3) Trajectories as mappings: We will assume in the following that trajectories are given as mappings from a compact interval of $\mathbb{R}$ to $\mathbb{R}^{3}$. The case of mappings from $\mathbb{R}$ to $\mathbb{R}^{6}$ (that is with explicit speed, for example as given by radar tracking filter [8], [9]) can be derived with minor changes and thus will not be addressed here. Since physical trajectories are smooth unless there is a perturbing noise, we made the choice to take all trajectories as smooth mappings from a compact interval of $\mathbb{R}$ to $\mathbb{R}^{3}$.

The first point to deal with is the necessary calibration of the origin of time for trajectories comparison. Remembering that there is an explicit dependence on time, one cannot just time shift one trajectory in time in order to make it coincident with another in order to compare them : this will result in forgetting distortions in time, that is trajectories with the same range (as mappings) but different positions at different times may become equal.

Since we choose to compare trajectories as mappings, a good candidate for computing the distance will be to integrate over time (like for the area distance) and to evaluate a mean error instead of the raw sum of squares :

$$
d_{T}\left(\gamma_{1}, \gamma_{2}\right)^{2}=\frac{1}{2 T} \int_{-T}^{T}\left\|\gamma_{1}(t)-\gamma_{2}(t)\right\|^{2} d t
$$

with $T>0$. This formula defines a semi-distance between trajectories $\gamma_{1}$ and $\gamma_{2}$.

The previous family of semi-distances has nice features because of the scaling ability, but since it is not a single metric, it is difficult to use standard algorithms based on distances (for example, classification algorithms). There is thus a need for another definition of proximity between trajectories that will yield a single value while capturing interesting global characteristics.

\section{B. New Distance Definition Based on Homotopy}

Before introducing our homotopic distance between trajectories one must introduce how do we cope with time difference between trajectories.

1) Parametrization invariance: The parametrization invariance is a very importante feature: the shape of an object is independent on the way its contour is followed. In its seminal paper, Kendall introduced the notion of shape manifold [16]: the originality of its work was the use of a differential geometry setting to implicitly enforce the invariance with respect to shape-preserving transformations. Curves were represented as finite sequences of distinguished points, called landmarks. Some related algorithms were eventually designed for air traffic analysis applications. In a study conducted by the Mitre corporation on behalf of the Federal Aviation Authority (FAA) [11], a spectral clustering algorithm was applied to sampled trajectories. Only the distance between landmarks was used, no invariance under euclidean transformations were imposed. Due to the high computational complexity, a random projection was first applied to the data in order to reduce the dimension of the samples. The most important limitation of this approach is that the shape of the trajectories is not taken into account when applying the clustering procedure unless a re-sampling procedure based on arc-length is applied: changing the time parametrization of the flight paths will induce a change in the classification. Methods based on times series as surveyed in [18], [24] are appealing, but are inadequate for the present application. Finally, functional data statistics [12], [22] provides a powerful framework, still lacking the re-parametrization invariance. In this section, flight paths will be modeled as points in an infinite dimensional riemanian manifold. In such space, each point is considered as a full trajectory. An intrinsic notion of distance exists in this setting and is defined as the infimum of the length of the paths connecting two points. Having this at hand allows the use of standard, distance based algorithms like k-means, k-mediods or hierarchical clustering.

2) Trajectories registration: A flight path may be modeled as a smooth curve $\gamma:[a, b] \rightarrow \mathbb{R}^{3}$ that maps a time to a position (at each time one can determine the associated position). Two distinct trajectories $\gamma_{1}, \gamma_{2}$ are most of the time defined on different time intervals, say $\left[a_{1}, b_{1}\right]$ (resp. $\left[a_{2}, b_{2}\right]$ ) for $\gamma_{1}$ (resp. $\gamma_{2}$ ), making the comparison between them quite awkward. This issue is well known in the field of functional data statistics as the registration problem. In a formal sense, it amounts to find a pair $\left(\phi_{1}, \phi_{2}\right)$ of strictly increasing diffeomorphisms $\phi_{1}:[0,1] \rightarrow\left[a_{1}, b_{1}\right]$, $\phi_{2}:[0,1] \rightarrow\left[a_{2}, b_{2}\right]$ such that the transformed curves $\gamma_{1} \circ \phi_{1}$, $\gamma_{2} \circ \phi_{2}$, defined on the common interval $[0,1]$, are as similar as possible. The special problem instance:

$$
\min _{\phi_{1}, \phi_{2}} \int_{0}^{1}\left\|\gamma_{1} \circ \phi_{1}(t)-\gamma_{2} \circ \phi_{2}(t)\right\|^{2} d t
$$

gives the Fréchet distance between $\gamma_{1}, \gamma_{2}$. Computing the optimal $\phi_{1}, \phi_{2}$ is a difficult task, unless the curves are assumed to be polygonal. Furthermore, as mentioned in [22], the registration procedure may remove some important features from the data: the extra degree of freedom provided by the so-called warping functions $\phi_{1}, \phi_{2}$ may have the detrimental effect of registering curves that does not need it [23]. A discrete relative to the Fréchet distance is known as dynamic time warping and may be used to compare sampled sequences. Nevertheless, it suffers from the same drawback (two trajectories having different time sampling will be condidered as different which is not relevent for our application).

3) Distance based on Homotopy between Trajectories: In order to compute the distance between two trajectories $\left(\gamma_{1}, \gamma_{2}\right)$, a time regularization is first applied to trajecto- 


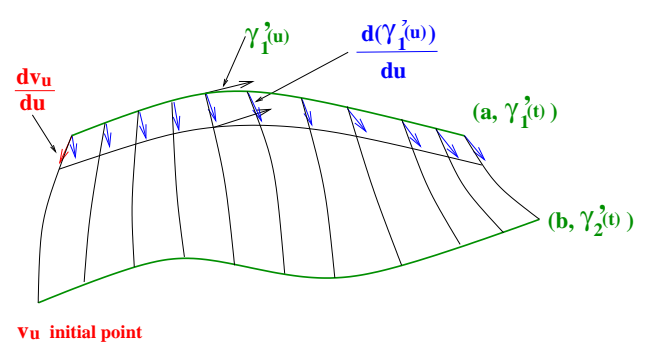

Fig. 8: Smooth path between two curves

ries. Then, an homotopy $\Phi$ between $\gamma_{1}, \gamma_{2}$ is built for which its associate energy is computed for extracting a distance metric (such distance has been developed by S.Puechmorel [21]).

Let $a$ be the origin of the trajectory $\gamma$. We have : $\gamma(t)=$ $a+\int_{0}^{t} \gamma^{\prime}(s) d s$, so a couple $\left(a, \gamma^{\prime}\right)(\in \mathcal{W})$ with $\gamma^{\prime}$ compactly supported defines a trajectory.

An homotopy between $\left(a, \gamma_{1}^{\prime}\right)$ and $\left(b, \gamma_{2}^{\prime}\right)$ is a continuous mapping $\Phi:[0,1] \rightarrow \mathcal{W}$ such that $\Phi(0)=\left(a, \gamma_{1}^{\prime}\right), \Phi(1)=$ $\left(b, \gamma_{2}^{\prime}\right)$. Intuitively, an homotopy is a continuous deformation between two trajectories (see Fig 8).

The deformation energy between $\gamma_{1}$ and $\gamma_{2}$ is linked to the distance between those trajectories and can be computed with the energy of the homotopy between $\gamma_{1}$ and $\gamma_{2}$ :

$$
E(\Phi)=\int_{0}^{1}\left(\left\|\frac{\partial v_{u}}{\partial u}\right\|^{2}+\int_{\mathbb{R}}\left\|\frac{\partial \gamma_{u}^{\prime}(s)}{\partial u}\right\|^{2} \cdot\left\|\gamma_{u}^{\prime}(s)\right\| d s\right) d u
$$

where $\frac{\partial v_{u}}{\partial u}$ is the normal vector (see Fig 8).

In order to compute such energy, a grid on the homotopy connecting $\gamma_{1}$ to $\gamma_{2}$ is built, as shown on Fig 9 .

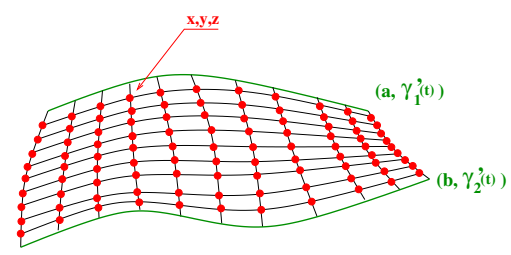

Fig. 9: Structure of the grid used for homotopy energy minimization. Each red point has 2D coordinates $(\mathrm{x}, \mathrm{y})$ for which an optimization algorithm is used for searching the $\mathrm{z}$ coordinates which minimize the energy of the homotopy connecting $\gamma_{1}$ to $\gamma_{2}$.

The optimization algorithm is searching for the $z$ coordinate of each grid point in order to minimize $E(\Phi)$. One can show that such problem is convex (from the optimization theory point of view) and gradient like method can be used to find the associated minimum (quadratic programming has been used to solved this problem efficiently). (a) On this metric space each trajectory is represented by a point (blue point).

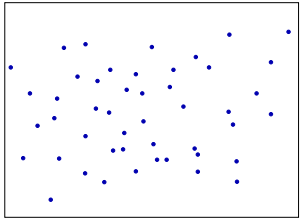

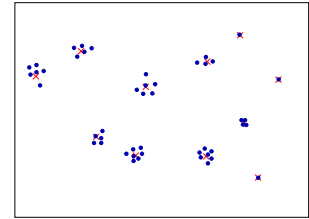

(b) In this example the algodifferent features. rithm find eleven clusters with

Fig. 10: Trajectory clustering algorithm representation

This distance can now be used in any distance-based clustering algorithm.

\section{Clustering Algorithm}

We consider a set of trajectories computed by the Bellman algorithm for each wind sample map. Having defined a distance between trajectories, one can gather together such trajectories in order to create clusters by using an adaptive clustering algorithm (hierarchical clustering). Such a clustering algorithm aims to partition the trajectory set into $K$ clusters. To reach this goal, trajectories are consider as points in the associated metric space (see Fig 10a).

This algorithm uses two parameters, $d_{\min }$ and $d_{\max }$, to respectively fuse clusters and create new clusters. Initially, each trajectory is considered as the centroid of a cluster. We then apply the three following principles one after the other:

- if two centroids are at a distance lower than $d_{\min }$, we fuse them into a single cluster, of which the resulting centroid is the barycenter of the two initial centroids.

- a new individual is aggregated to a cluster if its distance from the closest centroid is lower than $d_{\max }$ and in this case we compute the new global centroid.

- Otherwise, create a new cluster containing the single trajectory.

The number of clusters is also a result of the algorithm. An example of clustering result is given on Fig 10b.

For each cluster $c$, one can compute also the following features :

- Number of trajectories in the cluster $N_{c}$;

- Mean trajectory which is the cluster centroid $\left(\gamma_{c}\right)$;

- Dispersion of the cluster;

The overall processing on the trajectory clustering algorithm is summarized by the Fig 11

\section{RESUlts}

First we have consider two wind samples over the Atlantic ocean from two different days (January 09, 2016 and February, 14 2016). The first sample has few wind dispersion compared to the second one. An example of such map is given on Fig 12. 


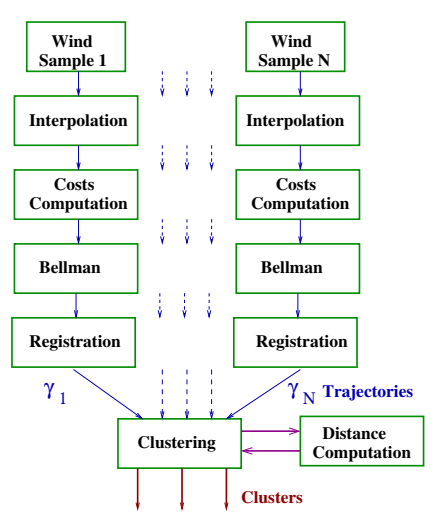

Fig. 11: Overall structure of the algorithm

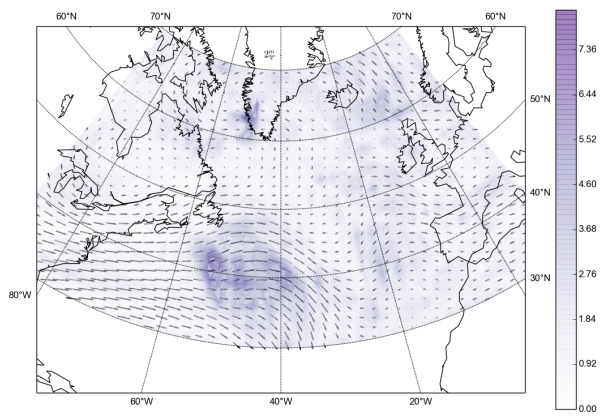

Fig. 12: Example of wind distribution over the Atlantic ocean

Those two days present different wind dispersion data with 34 wind samples at each point of the wind grid which (resolution : 1 degree).

Based on those wind data, we have applied the algorithm in order to compute the wind optimal route between two points. The origin has been settled at $\vec{P}_{o}=($ lat, long): $(\lambda=30$, $\theta=-90)$ and the destination at $\vec{P}_{d}$ (lat, long): $(\lambda=60, \theta=10)$. The first data sample presents less dispersion and may result in more robust planing. The Bellman algorithm has been applied 34 times between points $\vec{P}_{o}$ and $\vec{P}_{d}$ and has generated 34 trajectories that have been represented on Fig 13.

Those trajectories have been clustered by using the new distance that has been developed in section III-B. Two clusters have been extracted as it can be seen on Fig 14 . The first cluster has 30 trajectories and the second one 4 trajectories. The trajectories belonging to the first cluster are more robust and may be considered as the most robust wind optimal trajectories between $\vec{P}_{o}$ and $\vec{P}_{d}$. Fortunately, the best trajectory in terms of flight duration belongs also to cluster 1 (Flight time 11h34'; to compute this flight time,

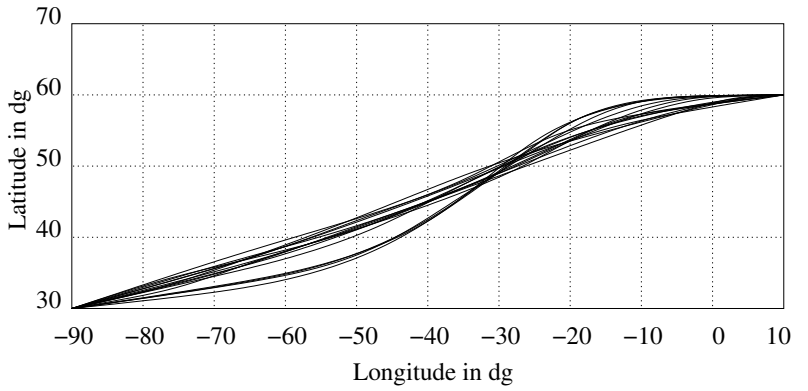

Fig. 13: Wind optimal trajectories for the first wind sample set (January 09, 2016). Each black curve corresponds to an optimal trajectory computed by the Bellman algorithm for each wind sample.

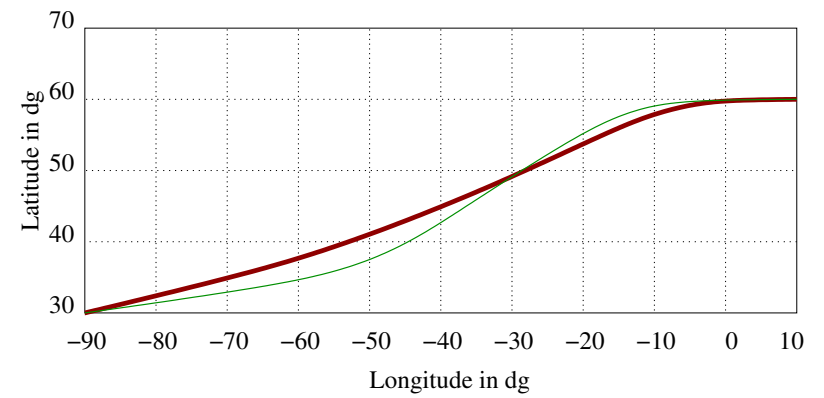

Fig. 14: Clusters produced for the first wind sample set. Two clusters have been extracted for which the most representative is represented in red. This cluster has 28 representatives and the other one has only 6 . This red cluster is then considered as the most robust trajectory.

a True Air Speed of 450kts has been considered). The best trajectory in cluster 2 has a flight time of $11 \mathrm{~h} 47^{\prime}$.

The second wind sample data from February, 142016 is more critical with more dispersion in the wind data and the trajectories produced by the Bellman algorithm are also more spread (see Fig 15).

Those trajectories have been also clustered and the associated cluster are represented on Fig 16. In this case, nine clusters have been extracted with a maximum of five representatives. In this case, there is not a big difference between cluster in terms of representative number, and we can say that the associated robustness is the same.

\section{CONClusion}

This paper has introduced a new approach for designing robust wind optimal trajectory. A methodology for computing tail wind on each link on a grid network over the Atlantic ocean has been introduced. First, wind has been interpolated on a more accurate grid, then tailwind formula on each link has been established and tail wind on each link has been computed. Based on this network an efficient 


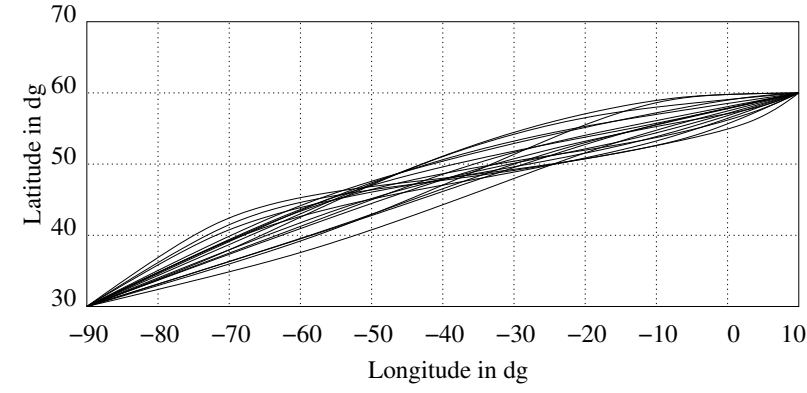

Fig. 15: Wind optimal trajectories for the second wind sample set (February, 14 2016).In this set, it can be noticed that optimal trajectories produced by the Bellman algorithm, present more dispersion.

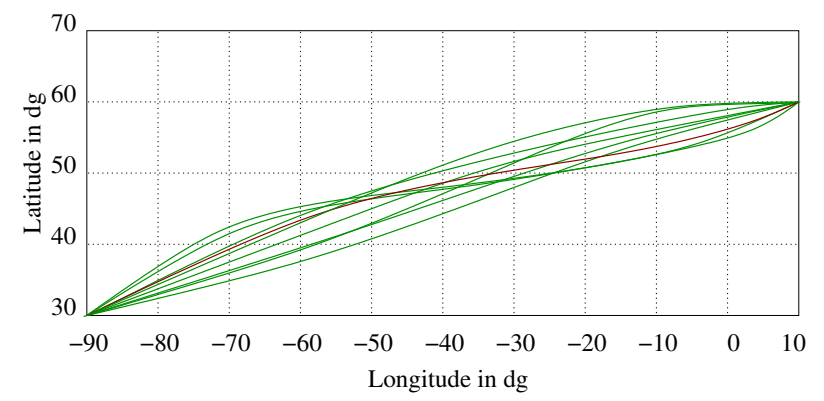

Fig. 16: Clusters produced for the second wind sample set. In this case, due to trajectories dispersion, nine clusters have been identified with near the same number of representatives(between 1 and three). The cluster with three representative (in red) may not be considered as robust and in this situation, it is very difficult to select one cluster among the others.

adaptation of the Bellman algorithm has been proposed thanks to the layers structure of the associated graph. In order to cluster trajectories produced by Bellman algorithm a hierarchical clustering algorithm has been developed and a new exact mathematical distance between trajectories has been introduced. Those algorithm have been successfully applied to real wind data in order to identify robust wind optimal trajectories.

As a future work, we will compare the associated performances of trajectories in terms of flight time and fuel consumption.

\section{REFERENCES}

[1] Arribas A, Robertson K.B, and Mylne K.R. Test of a poor mans ensemble prediction system for short-range probability forecastingensemble experiments on numerical weather prediction error and uncertainty for a north pacific forecast failure. Monthly Weather Review, 133(7):1825-1839, 2005.

[2] B. Arain and F. Kendoul. Real-time wind speed estimation and compensation for improved flight. IEEE Transactions on Aerospace and Electronic Systems), 50(2):1599-1606, 2014.
[3] A. Brezoescu, P. Castillo, and R. Lozano. Wind estimation for accurate airplane path following applications. In International Conference on Unmanned Aircraft Systems (ICUAS), 2013.

[4] Lu C, Yuan H, Schwartz B.E, and Benjamin S.G. Short-range numerical weather prediction using time-lagged ensembles. Weather and Forecasting, 22(3):580-595, 2007.

[5] Pêtrès C, Pailhas Y, Patron P, Petillot Y, Evans J, and D. Lane. Planning for autonomous underwater vehicles. IEEE Transactions on Robotics, 23(2):331-341, 2007.

[6] A. Cho, J. Kim, S. Lee, and C. Kee. Wind estimation and airspeed calibration using a uav with a single-antenna gps receiver and pitot tube. IEEE Transactions on Aerospace and Electronic Systems), 47(1):109-117, 2011.

[7] Shepard D. A two-dimensional interpolation function for irregularlyspaced data. In ACM Conf. ACM, 1968.

[8] D. Delahaye and S. Puechmorel. Tas and wind estimation from radar data. In IEEE/AIAA DASC. IEEE, 2009.

[9] D. Delahaye, S. Puechmorel, and P. Vacher. Windfield estimation by radar track kalman filtering and vector spline extrapolation. In IEEE/AIAA DASC. IEEE, 2003.

[10] Dijkstra E. A note on two problems in connexion with graph. Numerische Mathematlk, 1:269-271, 1959.

[11] M. Enriquez. Identifying temporally persistent flows in the terminal airspace via spectral clustering. In FAA-Eurocontrol, editor, ATM Seminar 10, 062013.

[12] F. Ferraty and P. Vieu. Nonparametric Functional Data Analysis: Theory and Practice. Springer Series in Statistics. Springer, 2006.

[13] Farin G. Level Set Methods and Fast Marching Methods: evolving interfaces in computational geometry, fluid mechanics, computer vision, and materials science, volume 3. Cambridge University Press, 1999.

[14] Hacker J.P, Krayenhoff E.S, and Stull. R.B. Ensemble experiments on numerical weather prediction error and uncertainty for a north pacific forecast failurea note on two problems in connexion with graph. Weather and forecasting, 18(1):219-260, 2003.

[15] Alton K. Dijkstra-like Ordered Upwind Methods for Solving Static Hamilton-Jacobi Equations. $\mathrm{PhD}$ thesis, The University of British Columbia, 2010.

[16] D. G. Kendall. Shape manifolds, procrustean metrics, and complex projective spaces. Bulletin of the London Mathematical Society, 16(2):81-121, 1984.

[17] C Lefas, C. Real-time wind estimation and tracking with transponder downlinked airspeed and heading data. IEEE Transactions on Aerospace and Electronic Systems), 23(2):169-174, 1987.

[18] T. Warren Liao. Clustering of time series data - a survey. Pattern Recognition, 38:1857-1874, 2005.

[19] C. Needham and R. Boyle. Performance evaluation metrics and statistics for positional tracker evaluation. In Proceedings of ICVS, Graz, Austria, pages 278-289, April 2003.

[20] Hart P, Nilsson N, and Raphae B. A formal basis for the heuristic determination of minimum cost paths. IEEE Transactions on Systems Science and Cybernetics, 4(2):100-107, 1968.

[21] Stéphane Puechmorel. Geometry of curves with application to aircraft trajectories analysis. Annales de la Faculté des Sciences de Toulouse. Mathématiques. Série 6, July 2015.

[22] J. Ramsay and B.W. Silverman. Functional Data Analysis. Springer Series in Statistics. Springer New York, 2006.

[23] J. O. Ramsay and Xiaochun Li. Curve registration. Journal of the Royal Statistical Society: Series B (Statistical Methodology), 60(2):351-363, 1998.

[24] Sangeeta Rani and Geeta Sikka. Recent techniques of clustering of time series data: A survey. International Journal of Computer Applications, 52(15):1-9, August 2012.

[25] M.E. Weber and M.L. Stone. Low altitude wind shear detection using airport surveillance radars. IEEE Aerospace and Electronic Systems Magazine), 10(6):3-9, 1995.

[26] X. Xiang, Z. Wang, and Z. et al. Mo. Wind field estimation through autonomous quadcopter avionics. In IEEE/AIAA DASC. IEEE, 2016. 